\title{
Tagesnachrichten und Persönliches
}

. Inter nation aler Dermatolog·en-Kongress.

Berlin, 12.-17. September 1904.

Der V. Internationale Dermatologen-Kongress findet laut Beschluss des IV. Internationalen Kongresses von Paris in den Tagen vom 12. bis 17. September $1904 \mathrm{zu}$ Berlin statt.

Anmeldungen von Yorträgen oder Demonstrationen werden spätestens bis zum 1. Juli 1904 an den General-Sekretär, Herrn Dr. 0. Rosenthal, Berlin W., Potsdamerstrasse 121 g, erbeteu. Da nur vier allgemeine Themata aufgestellt sind, kann den Vorträgen und Demonstrationen ein grösserer Zeitraum gewidmet werden, als dies auf früheren Kongressen möglich war. Ein kurzer Auszug des angemeldeten $\Lambda$ tortrags, am besten in Thesenform,. ist bis zum 1. Juli 1904 einzusenden, damit derselbe dem definitiven Programm beigefügt werden kann.

Der Mitgliedsbeitrag beträgt $20 \mathrm{Mk}$. (= 25 Frcs. = 1 Lstrl.) und ist an den General-Sekretär oder an den Landes-Sekretär zu entrichten.

Ehrenpräsident: Prof. Dr. E. Besnier, Paris. Präsident: Prof. Dr. E. Lesser, Berlin. GeneralSekretär: Sanitätsrat Dr. 0. Rosenthal-Berlin.

Themata:

Hautaffektionen bei Stoffwechselanomalien.

Syphilitische Erkrankuogen der Zírkulationsorgane.

Die Epitheliome und ihre Behandlung.

a) Stand der Verbreitung und der Bekämpfung der Lepra seit der ersten Leprakonferenz im Jahre 1897. b) Der gegenwärtige Stand der Lehre von der Lepra anaesthetica.

Prof. Dr. Jadassohn in Bern und Prof. Dr. P. V. Nikolsky in Warschau sind zu ordentlichen Professoren ernannt worden.

In Fiorenz hat sich Dr. F. Radaeli für Dermatologie und Syphili-graphie habilitiert. 